\title{
Acute Renal Failure in Tetanus
}

\author{
Satnam Kaur • Devendra Mishra • Monica Juneja
}

Received: 20 June 2012 / Accepted: 11 March 2013 / Published online: 29 March 2013

(C) Dr. K C Chaudhuri Foundation 2013

To the Editor: Despite being a preventable disease, tetanus remains an important public health problem in developing countries. Acute Kidney Injury (AKI) is a known, though not very well described, complication of tetanus; occurring in $15-39 \%$ cases. We report AKI and myoglobinuria in a child with tetanus, and discuss pertinent issues [1,2].

A 10-y-old boy was admitted with post-traumatic tetanus and was managed as per the unit protocol that included supportive care in an isolation area, tetanus immunoglobulin, intravenous Penicillin and Gentamicin, and diazepam infusion. He had severe muscle spasms for which diazepam infusion was gradually increased (upto $50 \mathrm{mg} / \mathrm{kg} / \mathrm{d}$ ) with sequential addition of chlorpromazine. His baseline renal parameters were normal. On day 4 of illness, he started having colacolored urine with decreased urine output. Investigations revealed myoglobinuria and deranged renal functions. There was no diurnal variation in blood pressure or any other signs of sympathetic over-activity. Urine output improved initially, in response to alkaline diuresis and furosemide, but, progressively decreased over the next $2 \mathrm{~d}(0.5 \mathrm{ml} / \mathrm{kg} / \mathrm{h})$. Peritoneal dialysis was started in view of rapidly rising blood urea (from baseline $32 \mathrm{mg} / \mathrm{dL}$ to $196 \mathrm{mg} / \mathrm{dL}$ ) and creatinine (from baseline $0.9 \mathrm{mg} / \mathrm{dL}$ to $7 \mathrm{mg} / \mathrm{dL}$ ), and persistent metabolic acidosis. Simultaneously, in view of persistent spasms, vecuronium infusion was started and child was put on bag and tube ventilation (due to non availability of ICU bed). However, few hours later, child had sudden cardiac arrest and died on day 6 of admission.

AKI has been previously described as a complication of tetanus. The renal dysfunction is reported to be mild and non-oliguric $[1,2]$. Suggested pathophysiological mechanisms include autonomic dysfunction, and rhabdomyolysis due to uncontrolled muscle spasms. Other contributory factors include nephrotoxic drug use, sepsis, and mechanical ventilation induced reduced cardiac output. Autonomic

S. Kaur $(\bowtie) \cdot$ D. Mishra $\cdot$ M. Juneja

Department of Pediatrics, Maulana Azad Medical College,

2, BSZ Marg, Delhi 110 002, India

e-mail: sk_doc@yahoo.co.in dysfunction, most evident in first two wk of illness, has been proposed to be the primary culprit in causing AKI [1]. No correlation was previously found between serum/urine myoglobin and ARF [1, 2]. However, no patient in these studies had gross myoglobinuria, and in the study that measured serum myoglobin, levels were below renal threshold, explaining apparent non correlation [3]. A case report describes anuric renal failure in tetanus, where onset of ARF was linked chronologically to elevations of CPK and myoglobinuria, similar to the chronological events seen in our case [4]. In another report of renal failure due to rhabdomyolysis and myoglobinuria, patient had a pelvic tumor and her baseline renal functions were deranged [5].

To conclude, AKI as a complication of tetanus occurs early during course of illness. Its prevention requires close monitoring to detect autonomic dysfunction, prevention of rhabdomyolysis by effective control of spasms, close follow up of urine output and renal parameters, and being watchful for myoglobinuria. The purpose of this communication is to increase awareness among pediatricians about this complication of tetanus, so as to take appropriate preventive measures and to recognize and manage it early.

\section{References}

1. Daher EF, Abdulkader RC, Motti E, Marcondes M, Sabbaga E, Burdmann EA. Prospective study of tetanus-induced acute renal dysfunction: Role of adrenergic overactivity. Am J Trop Med Hyg. 1997;57:610-4.

2. Martinelli R, Matos CM, Rocha H. Tetanus as a cause of acute renal failure: Possible role of rhabdomyolysis. Rev Soc Bras Med Trop. 1993;26:1-4

3. Mannix R, Tan ML, Wright R, Baskin M. Acute pediatric rhabdomyolysis: Causes and rates of renal failure. Pediatrics. 2006; 118:2119-25.

4. Asherson AP, Ruttmann TG. Severe anuric renal failure in a patient with tetanus. Br J Anaesth. 2002;88:740-1.

5. Weiss MF, Badalamenti J, Fish E. Tetanus as a cause of rhabdomyolysis and acute renal failure. Clin Nephrol. 2010;73:64-7. 